\title{
Management of Chronic Otitis Media (Safe and Unsafe Types) At Tertiary Care Center Rajivgandhi Institution of Medical Sciences (RIMS) ongole Prakasam District AP
}

\author{
Dr. K. Ravi M.S ${ }^{1}$, Dr. S. Kalyan Kumar M.S ${ }^{2}$ \\ ${ }^{1}$ Associate professor, ${ }^{2}$ Assistant professor
}

\begin{abstract}
Chronic otitis media is highly prevalent in the rural areas of Praksam district due to lack of awareness of ENT diseases, and illetaracy, and lack of sufficient no of Otologists .. and these case are common in childhood due to recurrent Upper respiratarory tract infections, adenoid hypertrophy, frquent rhinosinusitis. and these cases present highest no compared to other otorhino laryngological cases in department of ENT, RIMS Ongole. various surgical procedures, like Tympanoplasties (endoscopic and microscopic) and mastiodectomies either cortical or MRMs with or without cavity obliteration and ossiculoplasties for damaged ossicles are being done in the management of these cases..

Aim of the study: Study of various surgical procedures being done for chronic suppurative otitis media with tubo tymapic type with dry central perforation and with chronic mastioditis, ossicular necrosis and CSOM with attic perforation and with cholesteatoma, granulations polyps, retraction pockets. .This study was conducted in January 2016-2017 february. The main aim of this study isto make the ears dry, clean, safe ears and to prevent further complications, and further detioration of the pts and secondly to improve the hearing .

Material and methods: Study of CSOM cases in an year 2016 January to 2017 Febraury and 200 cases were selected and most of the cases were enrolled in NTR Vydya seva programme which is prestigiously being run by the Gov t of AP, and these cases are properly investigated and admitted and posted for surgical treatment after conservative management,
\end{abstract}

\section{Introduction}

Chronic suppurative otitis media with tubotympanic and attico antral diseases are quiet common in ENT opd at RIMS ongole ,they constitute $40 \%$ of total op ENT census. They present with either dry central perforation,polyps ,granulations,tympanoscerlotic patches,attic perforations,with cholesteatomas with or with constant purulent or mucopurulent discharge with mastioditis . Surprisingly these cases never presented with either extracranial or intracranial complications due to as they seek of medical treatment at early stage , and using higher antibiotics and they are coming to opd with presentation of ear discharge,hard of hearing,tinnitus and itching. .On examination most of these cases found to have central perforations of (either anterior,posterior,small,medium,and subtotal,total perforations), they have been investigated taking plane Xray of mastiod,audiograms to assess the hearing,pus swab from external auditory canal for culture and sensitivity for wet ears, CT mastoids, routine blood and urine examinations., and these cases were examined with oto endoscopy to confirm type of perforations, ,margins of the perforations,cleaning of the debris and finally these cases were admitted under NTR Vidyaseva programme for surgical procedures and after attaining the approval of the cases ,these were posted for surgery. For dry central perforations with intact ossicles Myringoplasty either Postaural approach or endomeatal approach with 0 degree endoscope the surgery was done. For those cases with mastioditis cortical mastiodectomy with TypeI tympanoplasty were done .For those cases presented with cholesteatoma Modified radical mastiodectomy .For those cased with limited cholisteatoma or retraction pockets combined approach mastiodectomy with intact canawall procedure done using both Endoscope and operationg microscope. For those cases with MRM cavity obliteration done using fibrofatty tissues or pedicle muscle flap,And all MRM cases aesthetic Meatoplasties were done. These cases were kept in the ward and discharged on the $7^{\text {th }}$ day and followed for 6 months with all post op videootoendoscopy images,postop PTA and Xray mastoids were documented.

\section{Surgical procedures}

These cases are posted for surgery under local anaesthesia, which is best for adults and cooperative cases and for children and incooperative pts general anaesthesia preferred. and all these cases after infiltration with $1 \%$ xylaocaine mixed with adrenaline, sedation given $1 / 2 \mathrm{hr}$ before the surgery with $1 / 2 \mathrm{cc}$ fortwin and with $1 \mathrm{cc}$ of phenergan intravenously slowly. These cases endoscopically meatal inscisons given to elevate the korners flap and standard post aural incision (wildes) given and Temporalis fascial graft harvested,and T shaped inscion given over the mastoid periosteum and flaps are elevated and pinna retracted and meatus with TM exposed, 
perforation edges are inscised and freshened and TM flap elevated ossciles exposed, if the middle ear mucosa healthy and dry graft kept medial to malleus,and if middle ear mucosa edematous and suspecting the pathology in antrum is suspected mastoid antrum opened with cutting burrs of various sizes and the patency of aditus checked by irrigation and graft is kept, and if there are granulations ,cholesteatoma debris present MRM done with bridge removal fascial ridge very much lowered upto incus or lateral semicircular canal, butteresses are very much reduced and necrosed ossicles are removed and reconstructed and if malleus incus are absent PORP kept and if all ossicles absent only foot plate is there then TORP kept, and in case of MRM cavities fibrofatty tissues along with muscle or perosteal flaps are reposited in the cavities for obliteration of the cavities, and wide meatoplasty done with removal of conchal cartilage. Depending upon the type of the disease, these procedures are being done using either with Endoscope, or microscope or using both with recording of all these procedures for documentation for NTR Vidyaseva programme which is prestigious in AP for those pts below poverty line.

\section{Statistical data}

ENT Cases of various diseases in OPD - 2016 January-2017Feb.

\begin{tabular}{|l|l|}
\hline No of pts visiting ENT op per day & 100 \\
\hline Tonsillitis cases & 10 \\
\hline Nonspecific throat pain & 15 \\
\hline Non specific Neck pains & 5 \\
\hline Nasal obstruction (DNS) & 14 \\
\hline CSOM & 30 \\
\hline Tubotympanic type & 25 \\
\hline Attico antral type & 5 \\
\hline Fb ear & 1 \\
\hline Tear ear lobule & 1 \\
\hline Malignancies & 1 \\
\hline Sinusitis & 8 \\
\hline Horseness of voice & 1 \\
\hline Reflux esophagitis/LPR & 2 \\
\hline Headache & 7 \\
\hline Neck swellings & One or non \\
\hline Dysphagia & 4 \\
\hline
\end{tabular}

ENT surgeries performed in 2016 Jan-2017 Febraury at RIMS Ongole

\begin{tabular}{|l|c|}
\hline No ENT surgeries performed per year & 840 cases \\
\hline No of ear surgeries & 360 \\
\hline Myringo plasties & 204 \\
\hline Mastiodectomies with tympanoplasties & 120 \\
\hline MRM with cavity obliteration & 36 \\
\hline
\end{tabular}

Grafical representation of ENT surgeries performed 1in 2016 Jan -2017 February

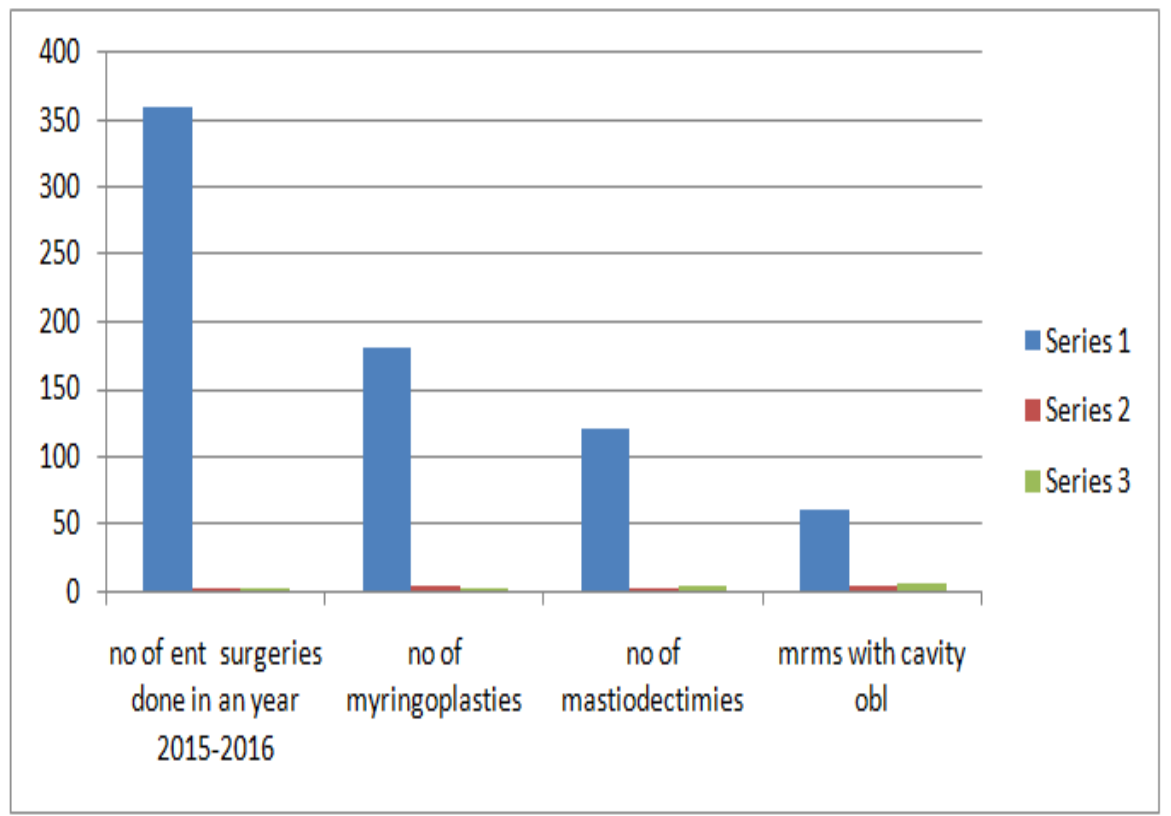

ENT OPD census in 2016 Jan -2017 Feb at RIMS Ongole 


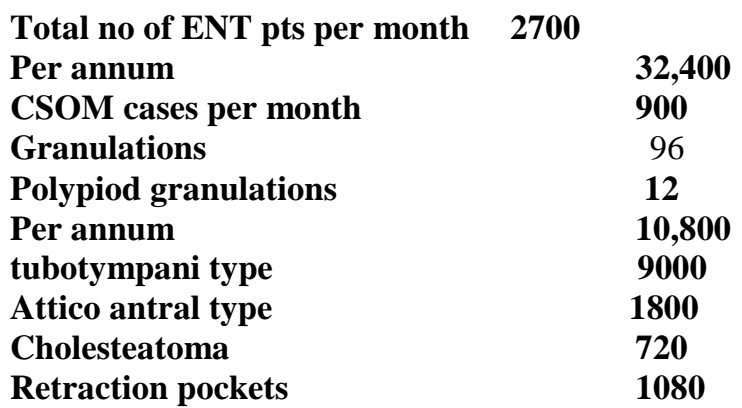

\section{Conclusion}

- Dry ear with Central perforation can safely be managed by Myringoplasty or Tympanoplasty.

- Cases with oedematous middle ear mucosa or with mastioditis can safely be managed by cortical mastiodectomy and with tympanoplasty with good results .

- Cases with attic defect ,cholesteatoma debris and attic granulations needs MRM and mastoid obliteration with reconstruction of ossicular chain.

- In about $95 \%$ of cases with attic defect, deep retraction pockets,granulations ,mastoid exploration revealed cholisteatoma.

- Proctor suggested the association of granulation tissue in $93-95 \%$ of cases of cholesteatoma. Retraction pockets were seen in few cases. $10 \%$ ossicular defects were seen in all cases.

○ In our study over all success rate was 96 (100 ears) 4 cases were advised revision surgery due to recurrent /residual disease

- In our study the minimum age of the pt was 10 years and maximum age was 55 yrs. Maximum no of pts i.e. $35 \%$ in the age group 11-20 yrs followed by $34 \%$ in the age group of 21-30 yrs

\section{Bibliography}

○ Black B Mastiodectomy elimination. Am J Otol19;551-557

- Gorur K,Ozcanc Unal M, (2002) Causes of failure in open cavity mastiodectomy

- Hoffmann DF,Fragan PA,Taylor B 1997 Reduction meatoplasty

- JacksonCG,schall DG,Glasscock ME3rd ,Macias JD, widick MH, a surgical solution for the difficult chronic ear.Am J Otol 17;7-14 (pubMed)

○ Mosher HP (1911) A method of filling the excavated mastoid with a flap from the back of the auricle. Laryngoscope 21;1158-1163.

- Ojala K, Sorri M , Sipilap,Palva A (1982) Late changes in ear canal volumes after mastoid obliteration . Arch otolaryngol 108;208-209 (PubMed)

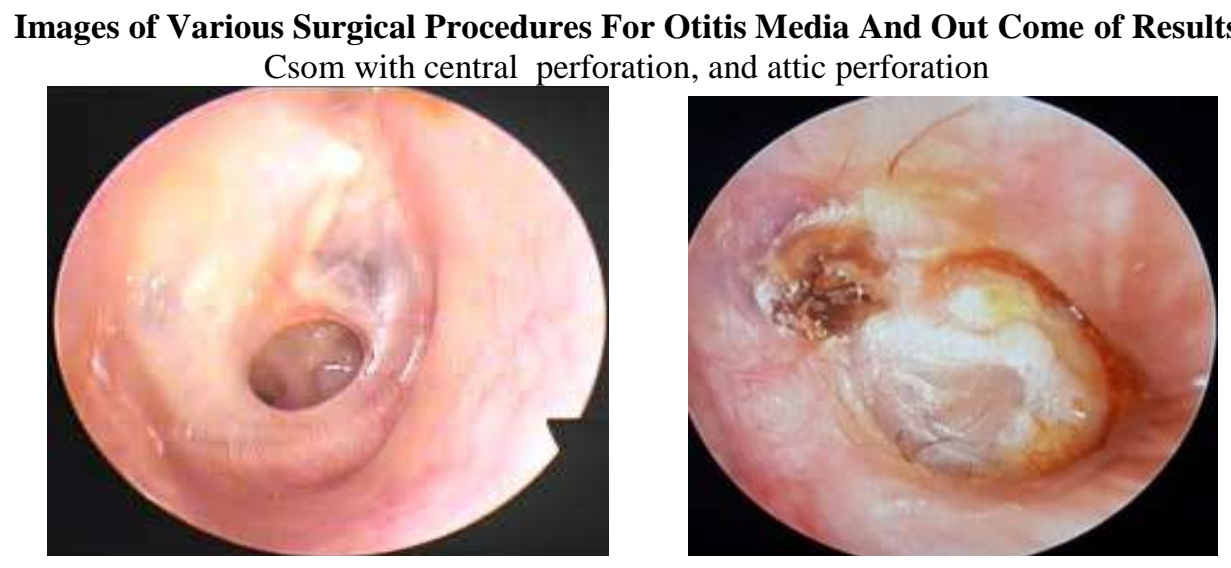


Postop MRM with cavity obliteration healed
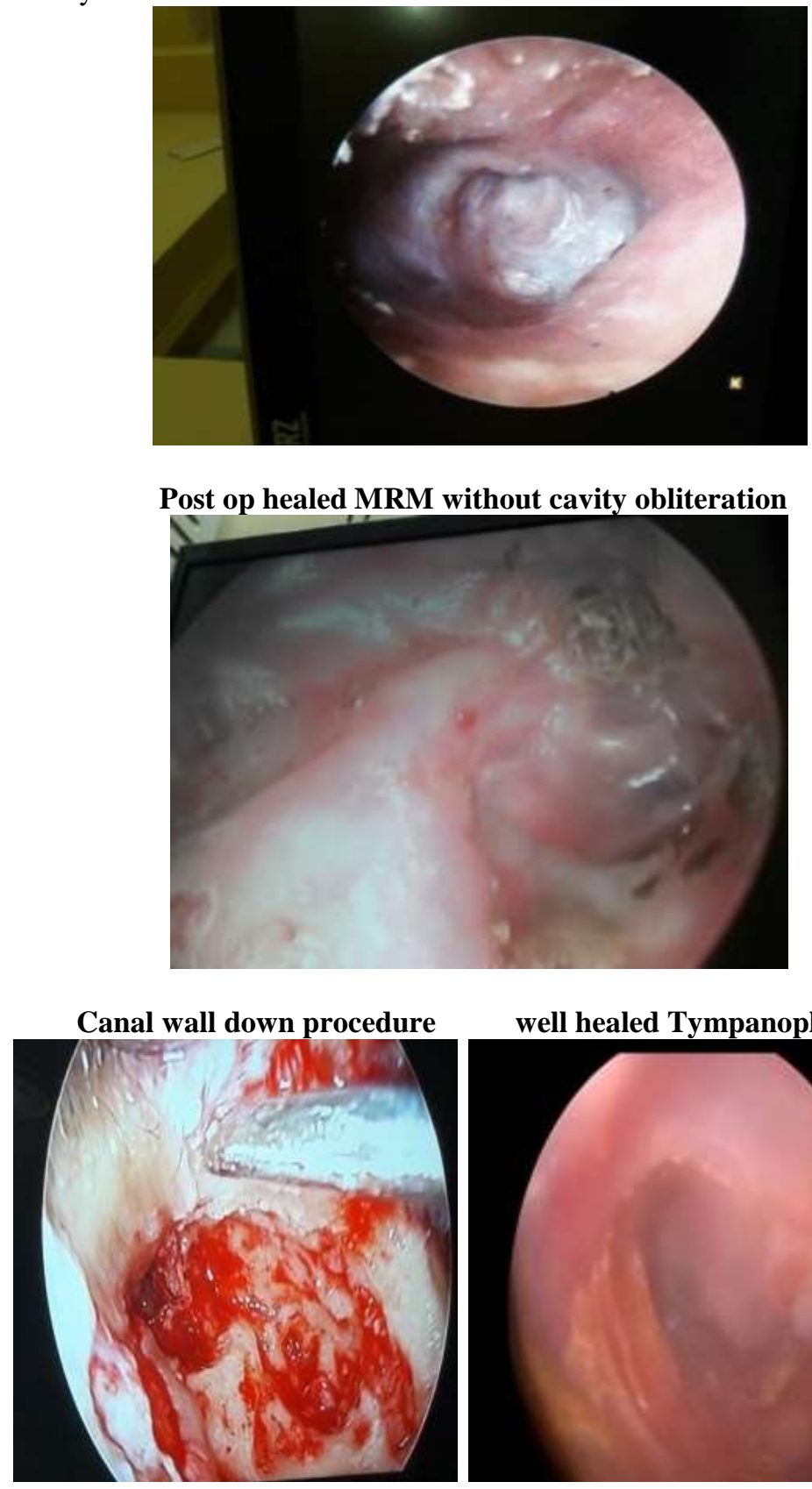

well healed Tympanoplasty

Ossiculoplasty

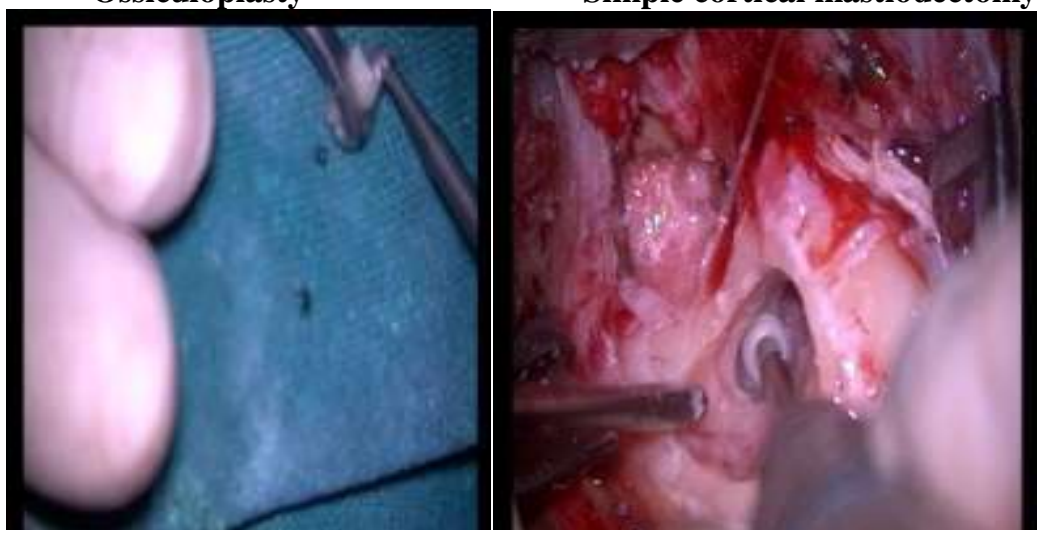

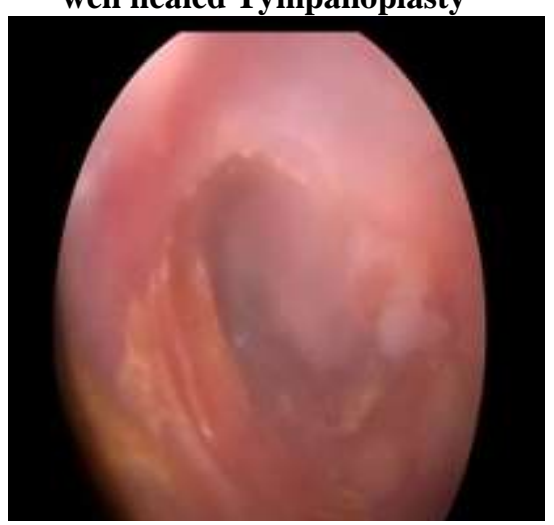

Simple cortical mastiodectomy 


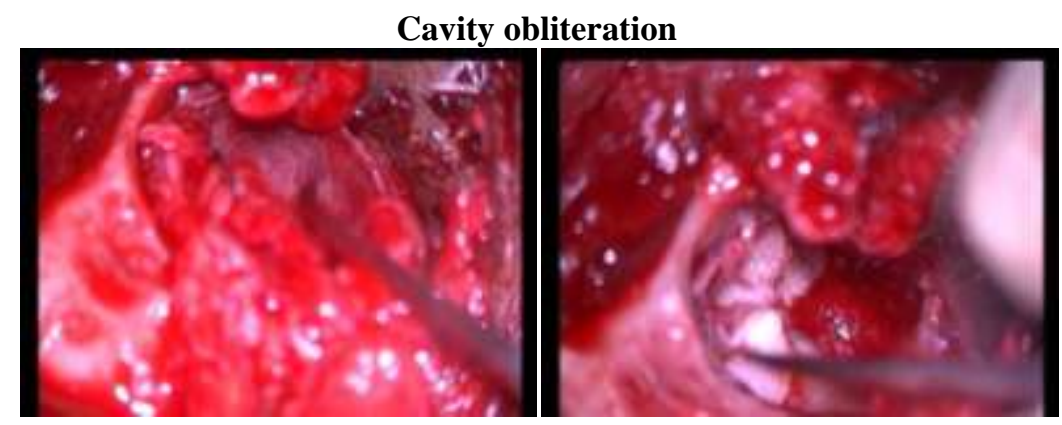

Cartilage slices augementation posterosuperior Meatoplastymeatal wall

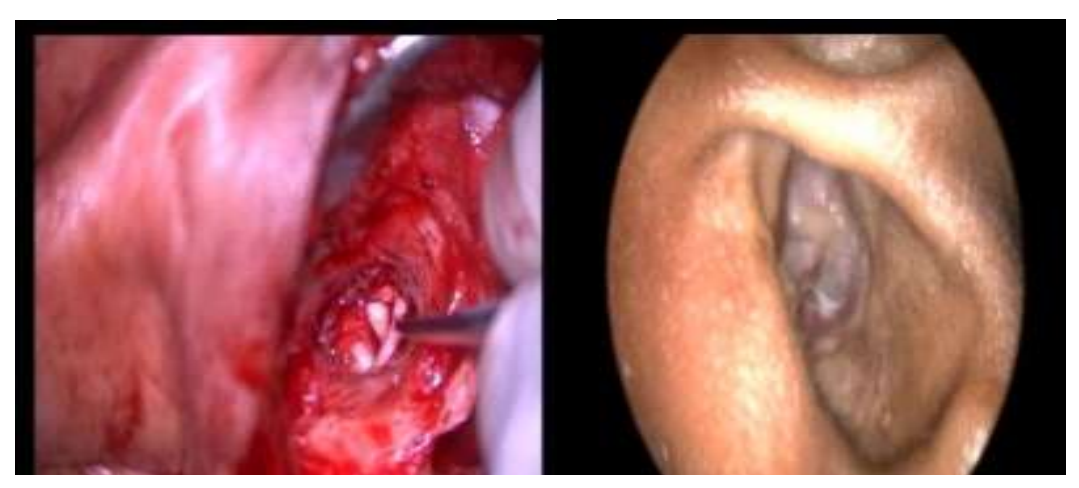

